\title{
Créativité littéraire et identité culturelle dans l'œuvre d'Ahmadou Kourouma
}

\author{
Alpha Ousmane Barry Laseldi \\ Université de Franche-Comté
}

\section{Position du problème de l'écriture francophone}

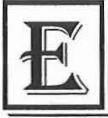

n nous appuyant sur la notion de configurations discursives ${ }^{1}$, nous nous proposons d'étudier une forme de trans-textualité qui apporte à l'écriture littéraire francophone d'Afrique des marques d'identités culturelles. La trans-textualité dont il s'agit dans cet article ne réfere pas aux phénomènes énonciatifs qu'on range dans les catégories habituelles de reprise du déjà-dit, du discours dans le discours ou du discours sur le discours. Mais il s'agit d'un phénomène qu'on peut désigner par l'expression discours social constitutif du discours littéraire. Ce qui revient à considérer le discours littéraire francophone d'Afrique comme un espace de négociation culturelle.

Partant de ce que le narrateur désigne par l'expression gros mots dans Allah n'est pas obligé d'Ahmadou Kourouma, par exemple, c'est-à-dire vocabulaire peu usuel et pas courant, voire grandiloquent, celui-ci se propose d'utiliser des dictionnaires pour chercher les gros mots, vérifier et surtout les expliquer parce que, dit-il, mon blablabla est à lire par toutes sortes de gens. La singularité de ce discours littéraire réside à la fois dans l'interconnexion de plusieurs langues et de plusieurs voix. Dès l'incipit d'Allah n'est pas obligé, le narrateur annonce, par exemple, l'utilisation de quatre dictionnaires, ce qui est l'indice de la coexistence dans l'écriture de mots issus de trois langues : le français de France, le français parlé en Afrique et le créole parlé au Liberia et en Sierra Leone, qu'il qualifie de pidgin. En dehors de ces langues, mises en contexte par un discours littéraire, l'écriture de Kourouma met en relation co(n) textuelle le dire et

1 Configurations discursives, Semen 8, 1993. 
le dit, la langue d'écriture et la langue maternelle de l'auteur. Cette écriture semble être le prisme où se reflète le discours social (Angenot 1989) ${ }^{2}$. Ainsi, sous des formes variées, la rumeur du discours social s'actualise dans l'écriture de Kourouma.

Proche de cette théorie du discours social se dessine le dialogisme chez Bakhtine $^{3}$. Selon cet auteur le discours romanesque résulte d'un immense travail de transformation systématique d'un ensemble verbal. Lequel est accompli par l'artiste dans le but de dépasser la frontière habituelle des mots pour construire une ouvre esthétique peuplée de plusieurs langages. Ainsi, la forme et le contenu constituent-ils un tout : le discours compris comme phénomène social. Cette conception du discours romanesque conduit à définir l'œuvre littéraire, comme une mise en scène dialogique. Mais si l'orientation dialogique est l'une des caractéristiques fondamentales de toute œuvre romanesque, elle est aussi un phénomène propre à tout discours. Le discours vit, en effet, en dehors de lui-même, dans une fixation sur son objet. C'est ce phénomène de dialogisme généralisé caractéristique de tout discours qu'on désigne habituellement par les vocables d'hétéroglossie ou de plurilinguisme du roman. C'est en cela que la sémiotique est transculturelle dans son essence et ses fondements. L'objet de cette étude se trouve définit par Semunjanga $\left(1999\right.$ : 28) ${ }^{4}$ de la manière suivante : la poétique transculturelle étudie comment les textes littéraires, qui renferment en eux des références aux catégories générales de la littérature (genres, thèmes ou motifs), mais aussi les catégories historiques (symboliques culturelles spécifiques), se différencient les uns des autres à partir des catégories en partie conventionnelles.

En concevant un champ d'étude du roman largement ouvert aux relations qu'entretiennent les productions culturelles issues de civilisations différentes, Semunjanga (1999) introduit la recherche de nouveaux concepts opératoires qu'il dénomme transculturalité et transgénérité. Selon l'auteur ces notions apportent un meilleur éclairage heuristique sur la nature du travail d'écriture en tant qu'activité de création comparable à la métaphore de l'abeille qui butine les fleurs pour préparer le miel. Ces

2 Marc Angenot, "Rhétorique du discours social ", Langue française, no 79, 1988, p. 24-36 et 1889 un état du discours social, Longueuil, Le Préambule, 1989.

3 Mikhaîl Bakhtine, Le Principes dialogique suivi des écrits du cercle de Bakhtine, Paris, Seuil, 1981.

4 Josias Semujanga, Dynamique des genres dans le roman africain, Paris, L'Harmattan, 1999. 
notions visent donc à étudier la relation transversale qui se négocie entre différentes productions culturelles dans une ouvre romanesque. En réponse à la question de savoir ce qu'on entend par poétique transculturelle, poétique transculturelle, Semunjanga (1999 : 19) avance que C'est la méthode d'analyse qui vise à montrer comment une ouvre artistique dévoile la culture de "Soi " et de l' Autre " par des coupes transversales sur les genres artistiques et littéraires. Elle veut dire de part en part des cultures et des genres littéraires, ou qui va à travers les cultures nationales et les genres, et aussi bien par-delà, au-delà de la culture et du genre. Il s'agit plus précisément d'une méthode qui étudie les relations quiune ouvre particulière établit avec la macrosémiotique internationale, trop riche et trop variée pour être dans le seul cadre national.

Le recours au conte, à la légende ou à l'épopée, etc. illustre mieux comment le discours romanesque travaille le feuilletage narratif en superposant les niveaux de l'énoncé et de l'énonciation. En se référant à la taxinomie des genres oraux en Afrique que propose Koné (1993), Barry $(2002,2004,2006)^{5}$ montre dans ses travaux ce qui relève des genres narratifs - le conte, l'épopée, la légende - et des genres non narratifs proverbes, dictons, devinettes, énigmes dans l'écriture d'Ahmadou Kourouma. Structures linguistiques stables coulées dans des syntagmes figés, les formes non narratives sont facilement identifiables. Au contraire, les substrats du conte, de l'épopée, de la légende et du mythe sont récupérés et coulés dans les canons du récit romanesque occidental. Dans ces conditions, il devient plus difficile de repérer dans le discours romanesque ce qui relève de la culture littéraire traditionnelle de ce qui est emprunté au roman occidental.

Sans pour autant se laisser emporter par une généralisation abusive, on peut dire que la plupart des auteurs africains de la seconde génération partagent la récupération et la réadaptation des genres narratifs traditionnels par le roman moderne. Ainsi, la création littéraire se présente comme une entreprise de destruction, de reconstruction ou de reconfiguration des genres narratifs traditionnels. Cela signifie que le texte fictionnel qui en résulte est calque du conte, la légende ou l'épopée. Au mieux, le remodelage de ces genres narratifs repose sur un jeu mimétique formellement identifiable pour peu que le modèle original soit perçu dans ses structures fondamentales.

5 Alpha Ousmane Barry, "Du mimétisme discursif au style dans l'entre-deux, un modèle de binarisme dans Les Soleils des indépendances d'Ahmadou Kourouma ", in L'oralité dans l'écrit réciproquement, CALS, 2002, p. 221-234. 
Comment, dans l'œuvre de Kourouma, la forme de l'écriture s'identifie-t-elle à l'espace de la mémoire mandingue? Comment le texte résultant de la création romanesque travaille-t-il à restituer le fond primordial par réminiscence ou mimétisme? Pour répondre à ces questions, nous allons, tout d'abord, dégager les grandes lignes du personnage épique et celui du conte, pour montrer ensuite en quoi le personnage dans l'œuvre de Kourouma présente des ressemblances avec celui des genres narratifs traditionnels.

Contrairement aux deux romans précédents où le substrat de l'épopée constitue la mouture de base pour la recréation littéraire, En attendant le vote des bêtes sauvages résulte d'un effet de réécriture du chant des chasseurs dans le milieu mandingue : le donsomana ${ }^{6}$. Le donsomana est une geste ou une ode qu'entonne le séréwa ou Sora au cours de veillées. L'énonciation de louanges qui rappellent les exploits de chasses s'accompagne d'une musique émanant d'un instrument à cordes : le nkoni. En mimant le chant de louange, une air propre aux chasseurs, l'auteur donne la parole au narrateur traditionnel - le Sora, griot appartenant à la confrérie des chasseurs, qui détient le monopole de la parole d'exhortation des chasseurs. Il domine incontestablement le récit romanesque, en maitre de la parole, il doit célébrer les gestes des chasseurs. Mais cette fonction exige quion parle du héros dès l'instant où son germe a été placé dans le sein de sa maman.

Dans sa narration, le Sora est accompagné d'une instance énonciative le cadoua qui joue le rôle de répondant. Il rythme l'énonciation du Sora par un namou, c'est-à-dire oui je t'écoute, comme cela se passe habituellement au cours des veillées de conte. Dès l'incipit, le narrateur principal, définit sa fonction de la manière suivante : Moi Bingo, je suis le sora; je louange, chante et joue de la cora. Un sora est un chantre, un aède qui dit des exploits des chasseurs et encense les héros chasseurs. Retenez mon nom de Bingo, je suis le griot musicien de la confrérie des chasseurs. (9). La fonction du Sora est cependant périlleuse, car l'élocution louangeuse doit faire corps avec la dénonciation du régime sanguinaire du dictateur. C'est à ce niveau qu'il met en pratique ses talents oratoires en maniant d'une façon très raffinée la parole. Le panégyrique du Sora jubile sous la forme d'un mélange burlesque de la langue de bois et des clichés traditionalistes. C'est un exercice d'habileté qui consiste à dire les vérités les plus terrifiantes en

6 Selon Dérive "Chez les bambara et les Maninka (Mali, Gambie, Guinée, Sénégal oriental), on a coutume de parler de donsomana, ce qui pourrait se traduire par "récits de chasse", à propos des champs épiques qui se rapportent aux différents épisodes glorieux de la vie des héros chasseurs " (1999 : 32). 
feignant la candeur la plus totale. Une fois investi dans ses fonctions de narrateur omniscient, il se déploie dans tous les sens et sa parole prend touts les dimensions. Le récit se transforme alors en déclamation poétique, un ensemble de scènes d'affabulation. Tel un panégyrique, la parole s'amplifie et s'étend aux langages et aux paraboles même apocalyptiques. L'écriture qui est à la fois espace total et parole achevée, dépasse les limites géographiques d'une territorialité fictionnelle pour investir mythe et légende. En occupant une large place dans la narration du récit, le Sora transforme le texte entier en donsomana. Dans ces conditions, le mimétisme discursif du récit oral n'est pas une technique de création romanesque implicite, mais au contraire, il constitue l'ossature même de l'œuvre.

En s'appuyant sur la production littéraire orale comme source de création romanesque, l'œuvre de Kourouma est, dans une certaine mesure, la réécriture de récits d'exploits de chasse, ce qui crée les conditions nécessaires d'attribution au personnage du président dictateur le statut de maître chasseur. Par la même occasion, la classe politique est considérée comme une confrérie de chasseurs. D'ailleurs le Sora s'adresse au personnage du président en ces termes : La politique est comme la chasse, on entre en politique comme on entre dans l'association des chasseurs. La grande brousse où opère le chasseur est vaste, inhumaine et impitoyable comme l'espace, le monde politique. Le chasseur novice avant de fréquenter la brousse va à l'école des maîtres chasseurs pour les écouter, les admirer et se faire initier. Vous ne devez, Koyaga, poser aucun acte de chef d'État sans un voyage initiatique, sans vous enquérir de l'art de la périlleuse science de la dictature auprès des maîtres de l'autocratie. (171)

Aussi avec l'assimilation permanente de la chasse et de la politique, la barrière entre l'humanité et la bestialité devient poreuse. On sait que cette frontière est déjà rompue dans Les Soleils des indépendances où le Parti Unique est comparé à une société de sorcières, où les grands initiés dévorent les enfants des autres. En levant les barrières entre animaux et humains, activité de chasse et pratique politique, activité productive et pratique occulte, la liberté formelle du roman se plie aux contraintes d'un chant ritualisé. Manœuvrant avec souplesse, l'auteur se laisse entraîner par son audace. La structuration du récit obéit au rythme de veillées à la place des chapitres. Chaque veillée est précédée d'un préambule, de trois proverbes, qui tient lieu d'exorde et se clôt par une série de trois proverbes : la péroraison. Le respect de ce rituel oratoire en fait une répétition qui construit un nouveau cycle emboîté en spirale. Ainsi la linéarité du récit est subvertie par des formes circulaires insistantes. Le récit s'organise en 6 veillées et 24 séances, 
comme si l'auteur s'était inspiré de la structure des contes de mille et une nuits. Chaque veillée s'organise autour des exploits de chasse d'un personnage qui entretient le double statut de chasseur et d'homme politique d'une part, et d'autre part de personnage traditionnel et de personnage du roman. Narrateur, personnages et discours qu'on leur attribue constituent alors un fond primordial de la culture source qui prend forme dans le roman moderne en processus de création. Tous ces éléments aident à la structuration du récit.

L'intrusion de ces formes esthétiques dans la littérature moderne met en lumière les mécanismes de continuité relative de la culture orale dans l'ordre scriptural. L'oralité feinte [dont parle Aliou Tine est donc] une espèce de caisse de résonance des différents récits, des différents types de discours et de langages qui circulent dans la société africaine. (Tine 1985 : 106) ${ }^{7}$. L'auteur souligne que l'ethno-texte et le discours de l'opinion sont l'espace où l'on peut lire l'identité de l'auteur et de son auditoire implicite (1985 : 113), c'està-dire la façon d'investir dans l'œuvre romanesque l'un des caractères essentiels de l'esthétique traditionnelle. Dans ce mélange de genres qui aboutit à la création de formes nouvelles, la régénération formelle ne consiste pas en une simple reconduction des techniques narratives de la culturelle originelle dans un genre d'importation.

La notion d'oralité feinte que propose Tine (1981) à propos de Sembène Ousmane trouve pleinement un terrain d'application dans l'œuvre d'Ahmadou Kourouma. Cette notion désigne en fait la tentative de l'écrivain africain de représenter dans son œuvre le narrateur traditionnel le Sora en l'occurrence - qui récite une histoire devant un auditoire. Le nouveau contrat énonciatif qu'institue le romancier n'est pas qu'oral, mais le passage de l'oral à l'écriture romanesque transite par la fiction. La représentation dans le roman moderne de narrateurs et de personnages traditionnels permet d'introduire des éléments narratifs de la culture mandingue. Loin d'être la transposition pure et simple d'une copie, la création romanesque assure la continuation entre le roman et les textes anciens. On peut déduire que le roman francophone est le prolongement de la parole artistique traditionnelle. L'examen des éléments narratifs et non narratifs ainsi que d'autres types de discours permet sans doute d'établir des liens d'affiliation entre roman africain francophone et genres littéraires oraux.

7 Aliou Tine, "Pour une théorie de la littérature africaine ", Présence Africaine, $\mathrm{n}^{\text {os }} 133-134,1981$, p. $99-121$. 


\section{L'ancrage du conte dans l'œuvre romanesque d'Ahmadou Kourouma}

On peut également avancer l'hypothèse que l'œuvre romanesque chez Kourouma joue aussi sur le rapport entre les traits génériques du roman occidental et ceux du conte africain. Cette hypothèse trouve son fondement dans le fait que la succession de certaines formes narratives rappelle celles du conte. Outre le fait que certaines séquences narratives insérées dans le récit romanesque proviennent de contes, le retour régulier de formules stéréotypées laisse penser aux chansons que scande le conteur au cours de son parcours narratif dans la plupart des contes populaires. On peut donc se poser la question de savoir comment les structures génériques du conte s'intègrent aux différents parcours narratifs de l'œuvre romanesque et comment elles établissent des rapports d'inclusion avec les motifs du genre romanesque occidental.

Dans l'œuvre d'Ahmadou Kourouma l'interférence entre deux narrations survient lorsqu'une séquence de conte intervient dans le récit ou, du moins, dans les cas d'imbrication d'un autre récit dans l'intrigue. Les mécanismes de mise en corrélation entre séquence de conte et récit romanesque se font à l'aide de traces énonciatives implicites ou explicites. Le lecteur des soleils des indépendances, par exemple, découvre facilement à la page 126 que les histoires de chasse permettaient de raccourcir facilement la journée. Cet indice atteste l'existence d'une séquence narrative du conte dans l'intrigue. Le chasseur Balla n'est pas un narrateur, mais il est le héros d'exploits de chasse. La narration de ses récits de chasse revient au griot Diamourou qui, en sa qualité de professionnel de la parole, est plus apte à restituer fidèlement les exploits de chasse de Balla. Ces récits qui sont racontés au second degré, car ils ne participent pas directement au récit premier, enrichissent cependant la diégèse. Dans Allah n'est pas obligé, au contraire, l'insertion d'une séquence de conte dans l'intrigue n'est pas explicite. Elle s'insère directement dans le texte et participe à la diégèse. L'extrait suivant peut, nous semble-t-il, servir d'exemple illustratif : Le matin commençait à arriver et nous continuons à marcher. Tout à coup, tous les oiseaux de la terre, des arbres, du ciel ont chanté ensemble parce qu'ils étaient tous contents, tellement contents. Cela a fait sortir le soleil qui a bondi vis-à-vis devant nous au-dessus des arbres. Nous étions contents nous regardions au loin le sommet du fromager du village quand nous avons vu arriver sur notre gauche un aigle. L'aigle était lourd parce quil tenait quelque chose dans ses serres. Arrivé à notre hauteur, l'aigle a lâché au milieu de la route ce quill tenait. C'était un lièvre mort. Tiécoura a crié de nombreux gros bissimilaï et a 
prié longtemps avec des sourates et beaucoup de prières de féticheur cafre. Il était soucieux et il a dit qu'un lièvre au milieu de la piste était très manvais, trop manvais augure (47). Le détour par la fiction, l'hyperbole, et l'imagination la plus débridée ont pour fonction esthétique de faire percevoir au lecteur la profondeur des souffrances vécues par les personnages. La critique sociale se dégage au terme d'un parcours fabuleux qui traîne le lecteur dans les méandres d'un récit dont les chemins sont parfois étriqués et sinueux. On peut déduire que le texte romanesque met en scène son propre simulacre à telle enseigne qu'il fonctionnalise sa lecture.

Des séquences entières de conte sont introduites d'une manière subtile sans qu'aucun indice explicite ne soit visible. La mise en corrélation narrative se fait sous le couvert de signes cosmiques de bon ou de mauvais augure que les deux compagnons - Birahima et Yacouba - rencontrent au cours de leur voyage. La manifestation de chaque présage donne l'occasion à Yacouba de mettre en pratique ses connaissances occultes en récitant des incantations ou une sourate coranique, voire les deux à la fois. Ce rituel incantatoire a pour conséquence immédiate d'éloigner le mal, de conjurer le mauvais sort ou bien de modifier le cours des événements. Cet épisode rappelle, d'une certaine manière, le combat que Balla le chasseur a livré au buffle génie dans l'intrigue des soleils des indépendances.

La septième séance de la deuxième veillée (82-95) dans En attendant le vote des bêtes sauvages fait le récit du coup d'état perpétré par Koyaga, tirailleur revenu du Vietnam contre le président de la République, brillant intellectuel. Le récit de ce coup d'état se présente comme un affrontement entre sorciers. Le modèle du récit est directement emprunté aux exploits de chasse narrés par le Sora. De façon plus lointaine une ressemblance frappante se profile entre le combat de Balla contre le buffle génie et le récit du coup d'état de Koyaga. Dans les deux cas les épisodes du combat sont magiques. Koyaga se transforme en un coq blanc puis il aveugle les gendarmes par la vertu de prières incantatoires. Fricassa Santos se protège en provoquant une panne générale d'électricité. Koyaga se transforme ensuite en oiseau nocturne muni d'une lampe. Enfin il se transforme successivement en fourmi puis en aiguille. Le président Fricassa Santos devient alors tourbillon de vent qui détourne les balles dirigées contre lui, mais il succombe aux flèches de Koyaga qui annulent sa protection magique. Au travers du récit de ce fabuleux combat, la figure du dictateur est saisie dans les trois étapes de son devenir : sa genèse, sa gloire, sa chute.

Cet épisode du récit entretient une ressemblance frappante avec celui de Balla, surtout quand on sait que livrant bataille au buffle génie, le 
chasseur balance son arme qui se maintient à hauteur d'arbre, entre ciel et terre. Le buffle se métamorphose en aigle et Balla en aiguille. Le buffle se transforme en fil rampant en direction du chas de l'aiguille. Le chasseur se métamorphose en brindille, le buffle se fait flamme. Enfin dans un ultime recours, le chasseur se transforme en rivière qui éteint la flamme. L'identité narrative de ces séquences de récits épiques montre comment la création romanesque repose sur un élément prédictif fondamental issu du récit héroïque traditionnel.

Outre ce mode d'introduction directe d'une séquence de conte dans le récit en cours, on peut remarquer que dès l'incipit d'Allah n'est pas obligé, le narrateur joue le rôle d'un conteur. Le récit commence par je commence à conter mes salades. Même si au lieu et à la place de conter l'auteur voulait dire raconter, quelques traces énonciatives recueillies dans le parcours de lecture rappellent le rituel du conte. On peut citer, entre autres, asseyez-vous et écoutez-moi. Et écrivez tout et tout (12). Enfin, on peut observer que mes salades rappellent aussi blabla, que le narrateur a élargi volontairement par une syllabe de plus pour donner blablabla, c'est-à-dire des paroles non sérieuses ou bien qu'on peut attribuer à de la fiction pure et simple. Cette voix populaire donne la représentation de la guerre civile dans un récit saisissant qui pourrait prendre le titre de voyage au bout de l'enfer.

La narration du récit dans Allah n'est pas obligé rappelle aussi le conte africain si l'on considère qu'une parole litanique parcourt tout au long du roman. Page après page le narrateur revient sur les mêmes paroles dites et redites dans un éternel recommencement. Ce mode énonciatif rappelle bien le travail du conteur, surtout quand on sait que la narration d'un conte est entrecoupée de séquences de chansons. La chanson comme parole rapportée a une fonction de mimésis. Elle renforce et accrédite le pouvoir du narrateur extradiégétique dont la distance apporte plus d'objectivité au récit. Lorsque des séquences de chansons sont insérées dans l'intrigue, elles ralentissent la narration et entretiennent ainsi plus de suspens dans le déroulement de l'intrigue. Les chansons ou leurs formes mimétiques sont convoquées à des moments décisifs qui coïncident avec le parcours narratif $\mathrm{du}$ personnage et de son compagnon d'infortune. En mimant ces chansons, l'auteur semble fondre l'intrigue dans une parole litanique.

Chez Kourouma l'œuvre romanesque est un espace redondant, mais dynamique du point de vue esthétique. L'écriture semble piétiner, or elle avance. L'extrait suivant semble être un exemple adapté à cette observation qui, au-delà de simples répétitions de mots, montre la reprise de groupes de mots ou de phrases entières de manière rythmique dans un style propre aux conteurs traditionnels africains. Parlant des bandits de grands chemins 
au Liberia et en Sierra Leone, le narrateur soutient, Ils se sont partagé la richesse; ils se sont partagé le territoire, ils se sont partagé les hommes. Ils se sont partagés tout... (53). Plus loin, on découvre également la répétition de prix cadeau en 6 occurrences dans un paragraphe. C'est pourquoi, [dit-il] on trouve tout à des prix cadeau au Liberia. De l'or au prix cadeau, du diamant au prix cadeau, des téléviseurs au prix cadeau, des pistolets prix cadeau [...] et tout prix cadeau (54).

Ces répétitions évoquent une mimésis de la chanson qui apporte du rythme dans le conte africain. Le retour régulier de formules stéréotypées ainsi que celui de certains énoncés donne à l'œuvre romanesque une densité singulière. Cette densité se reconnaît dans la syntaxe accumulative ainsi que dans les répétitions, ce qui conduit à parler de circuit de production et de reproduction discursive dans l'œuvre de l'auteur. Ce procédé d'écriture qui reproduit scènes narratives et séquences discursives montre que, comme le soutient Eco (1979: 63-64) ${ }^{8}$ un texte est un mécanisme paresseux (ou économique) qui vit sur la plus-value énonciative de sens qui y est introduite par le destinataire; ce n'est qu'en des cas extrêmes de préoccupation didactique ou d'extrême répression que le texte se complique de redondances et de spécifications ultérieures. On peut donc parler d'une sémiotique explicite du texte qui se caractérise par de multiples procédés de redondances et de feed-back.

Le substrat du conte se reconnaît aussi dans l'œuvre de Kourouma au profil du personnage de l'enfant-soldat qui semble à tous égards calqué sur un personnage du conte africain. Cette observation trouve son fondement dans le fait que la littérature traditionnelle accorde une attention particulière au statut de l'orphelin ou de l'enfant abandonné. Le personnage de l'orphelin quasi présent dans les contes introduit dans le récit les exactions de la marâtre, de la mauvaise coépouse, du mari irresponsable, du mauvais père, de l'injustice qui sévit dans les foyers polygames. Il développe des valeurs qui se rattachent à l'endurance physique et morale.

\section{L'ancrage des genres non narratifs}

Parmi les multiples références à l'oralité africaine, proverbes, devinettes, formules de salutation, paroles énigmatiques, maximes, dictons, adages, aphorismes sont certainement le substrat de la littérature traditionnelle qui se retrouve le plus dans l'ouvre romanesque francophone d'Afrique.

8 Umberto Eco, Lector in fabula..., Presses Universitaires de France, 1985. 
L'usage de ces énoncés savants soit par le narrateur, soit par le personnage principal, soit par d'autres personnages encore répond à un besoin didactique et/ou argumentatif. Ils véhiculent généralement une valeur morale. Contrairement à ces paroles qui énoncent une vérité, les prières, les incantations, les formules magiques, tout ce qui relève de la poésie liturgique est un domaine réservé aux seuls initiés : les sorciers, les marabouts, les maîtres initiateurs. Toute cette variété de discours se trouve imbriquée à la fois dans le discours romanesque, ce qui fait du roman un genre protéiforme. Notre travail consiste à observer la façon dont l'écriture sélectionne, retravaille et absorbe tous les éléments de la culture traditionnelle pour aboutir à une osmose qui fonde l'originalité du roman africain. Toutes les cuvres romanesques d'Ahmadou Kourouma sont profondément ancrées dans la culture mandingue. Outre l'influence des genres narratifs, l'ancrage des genres non narratifs est perceptible au niveau du tissu discursif dont l'architecture repose en grande partie sur des énoncés savants qui pavoisent le discours romanesque de formes ornementales. Au-delà de la seule fonction ludique, ces énoncés dégagent aussi une leçon de morale. Pour montrer la part grandissante occupée par les paroles savantes dans le discours romanesque, nous allons observer succinctement l'ancrage de ces énoncés doxiques dans deux romans de l'auteur.

En effet, si dans Les Soleils des indépendances le proverbe dépeint de façon métaphorique Fama. Il contribue à mieux camper son personnage en mettant en lumière ses défauts. Barry $(2002)^{9}$ indique dans ce contexte comment le proverbe arrive dans la conversation comme une vérité incontestable et illustre une situation précise, telle que le scandale provoqué par Fama. L'œuvre romanesque chez Kourouma apparaît ainsi comme une tribune de revendications culturelles et identitaires. L'écriture connecte des langues, des codes rhétoriques, narratifs et symboliques appartenant à des aires culturelles fort différentes. Elle établit des relais imprévisibles entre des mythes et des discours quotidiens dans une rencontre singulière faisant de l'œuvre romanesque un texte vivant qui mime la vie en Afrique dans toute sa complexité. L'aspect le plus remarquable de la création romanesque repose sur le fait que la régénération formelle n'est pas une simple reconduction des techniques narratives et non narratives empruntées à la culture originelle. Mais au contraire les catégories d'emprunt sont l'objet d'un travail de

9 Alpha Ousmane Barry, "Du mimétisme discursif au style dans l'entre-deux », op. cit., p. 221-234. 
déstructuration en profondeur. Elles sont retravaillées et redistribuées ensuite selon une combinatoire variable, de sorte que le passage de l'oral à l'écrit n'est pas senti comme une rupture, mais comme un continuum. Qu'en est-il dans les autres romans de Kourouma?

En parsemant une série de proverbes dans le discours du Roi, par exemple, l'écriture - dans Monnè, outrages et défis - tire des effets de style remarquables, comme le montre l'extrait suivant : Mais le dénouement des villages [...] L'indigence des gens [...] Les pays du Soba sont devenus exsangues. La limite de la bête est sa queue; il n'y a pas de forgeron qui à force de frapper, transforme le cuivre en or et aucun éreintement ne peut faire tirer l'eau de la pierre (110). Ces proverbes déploient des métaphores qui assument une fonction argumentative justifiant auprès de l'administration coloniale l'absence de bras valides volontaires pour mener la guerre contre les Allemands. Pour prévenir contre toute réaction violente de l'administrateur colonial, l'interprète parodie le discours du Roi lorsqu'il dit: La force comme lune est haute, elle ne peut suivre les soucis des minuscules fourmis perdues sur la terre. La ménagère arrange sur la claie de sa case les ustensiles selon leurs usages : près et à portée de la main les usuels; mais loin et haut, ceux qui réservés aux grands événements, qu'elle ne peut pas toucher qu'en hissant sur un escabeau. La guerre contre les "Allamas » est un grand événement, il faut monter sur l'escabeau pour atteindre ce qui n'est utilisé qu'exceptionnellement (110).

L'examen du contrat de communication qui unit le Roi, l'administrateur colonial et l'interprète permet de statuer sur les mécanismes de production du texte. La fécondation du texte par un langage métaphorisé et la traduction parodiée de l'interprète attestent que l'etho-texte n'est pas introduit tel quel dans le discours romanesque. Mais au contraire, il est subverti selon plusieurs modèles propres à chaque écrivain. La traduction trahie de l'interprète apporte un effet d'atténuation aux métaphores qui se dégagent des proverbes, lesquelles sont censées exprimer la force vitale du discours royal. Il en résulte une parabole coulée dans un langage euphémique dont le vocabulaire courant renvoie aux activités domestiques des femmes. L'usage des proverbes dans un contexte bien déterminé et leur introduction dans le nouvel univers romanesque à l'issue d'une transformation montre bien là où intervient la main de l'artiste.

On peut déduire que l'ethno-texte est le support qui féconde l'instinct créateur de l'artiste. Les proverbes qui constituent une des composantes de la culture traditionnelle ont pour effet pragmatique de frapper l'imagination du lecteur. C'est pour cela que la plupart des auteurs africains 
les utilisent pour insuffler dans le récit une surdétermination de sens. Le poids qu'apportent les expressions de structures parfois rythmiques est fortement travaillé par leur contexte d'emploi en vue d'un réinvestissement symbolique. Il rajeunit l'écriture par l'enchâssement de nouvelles catégories esthétiques, ce qui apporte à l'œuvre romanesque une force motrice.

De tous les romans d'Ahmadou Kourouma, on peut avancer qu'En le vote des bêtes sauvages est celui qui renferme le plus de proverbes. La mise en vedette énonciative et rhétorique du proverbe en fait l'exorde et la péroraison de 6 séances de 24 veillées. En tant que cheville ouvrière de la parole, le proverbe rythme de manière singulière le donsomana. Borgomano $(2000)^{10}$ souligne que la seule table des matières compte 24 proverbes correspondant à 24 séquences du chant. Chacun de ces proverbes est le premier d'une suite de 3 proverbes, comme nous l'avons souligné précédemment. 72 proverbes sont regroupés autour de la thématique de chaque veillée. On peut citer en exemple l'extrait suivant : Et quand on ne sait pas où l'on va, quion sache d'où l'on vient (11), On tarde à grandir, on ne tarde pas à mourir (72), C'est celui qui ne l'a jamais exercé qui trouve que le pouvoir n'est pas plaisant (169), Il n'y a pas de mauvais roi mais de mauvais courtisans (225), Au chef il faut des hommes et aux hommes un chef (250), Le jour éloigné existe mais celui qui ne viendra pas n'existe pas (338).

Outre ces proverbes situés au premier plan par leur fonction rituelle d'ouverture et de fermeture de chaque récit de chasse, de très nombreux autres proverbes, dictons et adages donnent au panégyrique du Sora une densité singulière. C'est en cela qu'on lui reconnaît sa profession de maittre de la parole. L'emploi des proverbes par le Sora se justifie par le fait que ces énoncés participent au balisage de la parole, comme s'ils la rendaient plus pesante. Le choix stratégique de tels outils, en tant que moyens rhétoriques est largement tributaire de la richesse du répertoire et de la mémoire discursive du Sora. Borgomano (2000) évalue leur nombre à plus d'une soixantaine, que l'auteur élargit d'ailleurs aux comparaisons qui fonctionnent sur le même registre. En général, la fonction sémiotique des proverbes repose sur les métaphores qui s'organisent dans le roman par groupe thématique. Tout comme pour les fables de la Fontaine, le symbolisme animal est très marqué au niveau de tous les proverbes. Ces formes métaphoriques qui font appel au symbolique animal sont coulées dans un langage poétique dont la forme esthétique repose en grande partie

10 Madeleine Borgomano, Des hommes ou des bêtes? Lecture En attendant le vote des bêtes sauvages d'Ahmadou Kourouma, Paris, L'Harmattan, 2000. 
sur sa structure binaire. Ce jeu de sonorités frappe l'attention et contribue fortement à entretenir le plaisir de lire le texte. La mise en spectacle du proverbe dans le récit du Sora installe les auditeurs dans un univers de croyances qui correspond aux imaginaires sociaux. La séduction qu'apporte l'usage du proverbe par l'orateur est un masque qui empêche l'auditeur de percevoir le projet de parole qui se trame en dessous. L'esprit de l'auditeur est séduit par le plaisir que lui procure un énoncé savant et il se trouve enfermé dans un cercle discursif. $(2002: 231)^{11}$.

Nous avons déjà abordé dans le cadre des genres narratifs la place du chant dans le conte. Le rythme énonciatif du Sora est aussi entrecoupé de chansons. On sait que le récit de chasse entretient un lien génétique avec le conte. La ressemblance entre les exploits de chasse de Balla et la bataille qui a opposé Koyaga à Fricassa Santos en est un exemple frappant. Mais contrairement au proverbe qui rythme le panégyrique du Sora, et que l'on retrouve dans tous les compartiments du discours romanesque, les chants font leur entrée surtout dans la veillée 5 qui comporte quatre couplets d'hymne de chasseurs. Il s'agit du chant des coqs de pagode (294), celui des jeunes chasseurs, c'est-à-dire l'hymne de l'aigle royal réservé aux seuls initiés (295), la voix du tambour des grands chasseurs (295) et le djandjon, hymne de la vaillance ou hymne des grands empires et des grands événements (295-296). Cet hymne invoque aussi le couple Kointron et Sanéné, ancêtres des chasseurs. L'existence de ce couple imaginaire s'origine dans le mythe fondateur de l'activité de la chasse.

On peut déduire de la rencontre de cette mosaïque de genres discursifs oraux la fragmentation incessante de l'axe syntagmatique qui rompt la linéarité du récit romanesque. Cela revient à reconnaître que les techniques de la création romanesque francophone d'Afrique reposent en grande partie sur la manipulation de la discontinuité formelle du récit et sur l'intégration narrative des substrats narratifs et non narratifs de l'ethnotexte. Ainsi le roman récupère-t-il l'oxygène du paradis perdu afin de recréer un monde moderne plus respirable.

\section{Parole énigmatique, discours crypté : fiction et réalité}

L'œuvre romanesque chez Ahmadou Kourouma entretient dans tous ses compartiments l'ambiguïté entre fiction et réalité, entre l'allusion à des

11 Alpha Ousmane Barry, "Les proverbes, un argument d'autorité dans l'art oratoire chez Sékou Touré ", in Pouvoirs du discours et discours du pouvoir L'art oratoire chez Sékou Touré de 1958 à 1984, Paris, L'Harmattan, 2002. 
personnes réelles et la mise en action de personnages fictifs. De cet amalgame, le narrateur tire un récit coulé dans un discours satirique qui entraîne le lecteur dans une illusion parfaite. Cette observation trouve sa validité dans le fait que tout ce qui est décrit dans le roman est si proche du quotidien que cela paraît vraisemblable. Mais avant d'aborder cet aspect de l'analyse sémiotique plus en profondeur, nous allons tout d'abord présenter ce que nous entendons par parole énigmatique.

Si les prières, incantations formules magiques, poésie liturgique sont dites par les seuls spécialistes, la parole énigmatique apparaît essentiellement dans la bouche des sorciers, des devins, des marabouts, personnages traditionnels que l'on retrouve habituellement dans le roman francophone. Cette parole est une interprétation de présages cosmiques, de cris d'animaux, de consultations du devin ou du marabout. Elle peut se présenter sous la forme d'un rêve prémonitoire. Dans Les Soleils des indépendances, les hurlements de la vieille hyène de Togobala sont une parole énigmatique qui s'interprète. On sait qu'elle avait annoncé : une ancienne et grande chose sera vaincue par une autre ancienne et grande chose (187). L'hyène annonçait ainsi la mort prochaine de Balla le chasseur qui était à la fois le plus âgé de Togobala et le plus craint des villageois à cause de ses pouvoirs magiques et maléfiques. Le personnage se faisait passer ainsi pour une personne immortelle. Aussi, la voix qui s'adresse à Bakary, l'ancêtre de Fama, s'adresse à celui-ci par énigmes. Elle lui prédit que s'il acceptait la chefferie, sa descendance coulerait, faiblirait et sécherait un jour où le soleil ne se couchera pas, où des fils d'esclaves, des bâtards, lieront toutes les provinces avec des fils, des bandes et du vent, et commanderont (101102). Cette parole que Bakary n'est jamais arrivé à décoder devient tout à fait claire pour Fama. Une ville où le soleil ne se couchera pas (les lampes électriques éclaireraient toute la nuit dans la capitale), où les fils d'esclaves et les bâtards commandent, triomphent en liant les provinces par des fils (le téléphone), des bandes (les routes) et le vent (les discours et la radio) (102).

La parole énigmatique s'inscrit toujours dans le cadre d'un récit. Le récit fondateur de la dynastie des Doumbouya présentée en ces termes constitue une composante de premier plan de l'intrigue romanesque. Elle infléchit le cours des événements et exerce un effet de fatalité sur le destin du personnage principal. Les prédications du marabout Abdoulaye transmises à Salimata s'inscrivent aussi dans le cadre des paroles énigmatiques. Il n'est pas inintéressant de souligner la façon dont la croyance est utilisée par le romancier moderne. La parole énigmatique remplit le même rôle que celui des autres récits traditionnels. Elle est énoncée pour être décodée, elle prédit l'avenir ou recouvre le sens caché. 
Avec sa double fonction esthétique et idéologique, le mythe moulé dans la parole énigmatique est un document d'une valeur ethnologique certaine. C'est pourquoi elle entre dans la logique de la fiction racontée.

L'œuvre romanesque de Kourouma fonctionne en grande partie sur la base d'un discours crypté. Ce cryptage intervient quand la satire déploie ses batteries pour dénoncer les abus, la violence, l'iniquité, caractéristiques des régimes qui ont vu le jour dans la période postcoloniale en Afrique. Le récit met en scène le sens de l'histoire africaine telle qu'elle est vécue au quotidien à travers le pouvoir politique des pères des indépendances sous la direction du parti unique, des dictatures militaires et des démocraties qui ont enfanté les guerres tribales et les rébellions. Avec Les Soleils des indépendances, l'auteur aborde l'histoire immédiate, celle des premières années d'indépendance. Dans Monnè, outrages et défis, Kourouma reprend l'histoire à partir des conquêtes coloniales jusqu'à la veille de l'indépendance. Dans En attendant le vote des bêtes sauvages, l'histoire commence par le rappel du partage de l'Afrique ${ }^{12}$ entre les puissances européennes. Le récit ménage des épisodes qui concernent la période d'indépendance, la guerre froide et même les turbulences de la fin de cette époque. Dans Allah n'est pas obligé, le début du roman entretient une ambiguïté au niveau des repères temporels. Le roman commence avec l'enfance du personnage principal âgé de 12 ans. Toutefois le récit fait référence à des événements ayant des repères dans la période coloniale. Mais en réalité tout se passe entre la fin des années 80 et la décennie de 90 .

Kourouma travaille à établir une interconnexion récit romanesque/ récit historique. Dans cette perspective, le roman de Kourouma peut servir de source pour les études historiques sur l'Afrique. C'est un ensemble de représentations qui permet de reconstruire la réalité. Il allie la trame narrative du conte, de la légende, de l'épopée, aux mécanismes historiques qui conditionnent la vie quotidienne du peuple. Aux personnages reconnus par la tradition historique sont adjoints des personnages purement imaginaires, d'où la tendance à considérer que tout en racontant le monde qui l'entoure ou son extériorité, l'œuvre romanesque est aussi une réflexion sur le processus qui l'engendre. Autrement dit le roman reconstruit le climat d'une époque. Telle une histoire romancée, l'énonciation romanesque réfléchit sur l'art de la création romanesque et sa parenté génétique proche ou lointaine avec l'histoire. Ces considérations

12 Cet événement historique, qui annonce la fin de la traite des esclaves et le début de la colonisation en Afrique est connu sous le nom de la conférence de Berlin en 1884. 
conduisent à réfléchir sur la plénitude référentielle et l'esthétique de la représentation romanesque. On sait d'avance que l'œuvre d'Ahmadou Kourouma s'inscrit dans la période des grandes turbulences politiques et sociales de l'Afrique. On peut donc parler, chez cet auteur, d'une esthétique de l'engagement politique et social pour l'avènement d'un monde meilleur.

L'extrait suivant est un exemple adapté du jeu qui se construit tout au long du récit romanesque entre la réalité historique et la fiction. Il concerne le récit de la fin de la deuxième guerre mondiale que Soumaré interprète $\mathrm{du}$ Roi Djigui. Tout commence par le commandant Héraud parla longtemps; l'interprète traduisit; pour la compréhension du centenaire, le griot commenta et interpréta les derniers événements intervenus dans le monde pendant que Balloda vivait des saisons d'amertume.

À la suite de l'extrait précédent qui donne une idée sur la place de l'interprète dans le récit en cours, l’infructueuse tentative de débarquement à Dakar ne découragea pas le général De Gaulle. Bien au contraire. Il monta et rencontra ses trois autres collègues. Ils se réunirent à quatre, les quatre grands parmi les cinq qui sétaient partagé le monde. Lui, De Gaulle, chef des empires du sud (les Arabies, les Négrities et les mers australes), Churchill, chef des empires du Nord (Londres, les îles britanniques et tous les océans nordiques), Roosevelt, chef des empires de l'Ouest (New York, les Amériques et les océans du couchant), Staline, chef des empires du Levant (Moscou, les Russies et tous les océans orientaux). Eux, les maîtres des quatre points cardinaux, jugèrent de poursuivre la guerre et de ne l'arrêter que le jour où ils auraient détruit le cinquième empire et tué Hitler, cinquième mầtre du monde, chef des empires du milieu (Berlin, les Frances, les Italies et les mers du Milieu). Les quatre alliés s'en allèrent consulter le plus grand devin de l'univers qui leur dévoila les secrets de guerre du maître de Berlin, ses totems, ses faiblesses et leur recommanda des ensorcellements quils pratiquèrent, des sacrifices quïls égorgèrent. Après les libations et les sacrifices, de Gaulle descendit à l'extrémité des Négrities à Brazzaville, y rassembla les Nègres de toutes les tribus, dont ceux de Soba. Il constitua une armée redoutable à la tête de laquelle il remonta le désert par les pistes des pèlerins, feignant d'aller au pèlerinage de la Mecque (215-216).

Cette séquence du récit montre comment l'art de la création romanesque chez Kourouma consiste à raconter des épisodes anecdotiques, en installant grâce à l'humour une convivialité dans le texte. L'humour s'appuie sur le détournement et la reconfiguration de la réalité historique. Contrairement à l'ironie mordante, l'humour chez Kourouma invite le lecteur à participer au plaisir du texte. Les personnages de l'histoire contemporaine évoqués dans l'extrait précédent, la référence à la deuxième guerre mondiale, la conférence de Brazzaville sont autant de réalités 
conformes à la vérité historique. Il existe donc une conjonction permanente entre réalité historique et fiction romanesque. D'ailleurs trois époques s'emboîtent constamment dans l'univers romanesque chez Kourouma : l'histoire de Samori, l'ère coloniale et les indépendances. L'allusion à des faits historiques se rapportant à ces époques est parfaitement intégrée dans le processus de la narration. Cela conduit à admettre que toute l'histoire coloniale et celle de l'indépendance est relue à la lumière de la métaphore de la collaboration. Si la référence à ces périodes historiques est importante, pour qu'on s'y attarde au cours de l'analyse, le plus intéressant ce n'est pas tant la référence à une réalité sociale, que le mimétisme discursif ou l'imitation des langages sociaux.

L'extrait précédent est un exemple de travestissement des faits historiques. Que De Gaulle, Churchill, Roosevelt, Staline, Samori, aient existé, cela ne fait l'objet d'aucun doute. Mais que ces figures historiques aient consulté un devin avant de livrer la guerre à Hitler ou bien que De Gaulle ait eu l'intention d'effectuer le pèlerinage à la Mecque, cela crée un décalage entre le récit et l'histoire qui s'intègre dans le cadre d'une pure invention romanesque. Cette valeur ajoutée qui relève du burlesque apporte au récit une valeur humoristique. C'est en cela que le regard distancié du romancier sur la réalité nourrit l'humour avec verve. Au contraire, lorsque le récit fait allusion à l'ordre donné à Djigui roi du Soba de détruire sa capitale avant l'arrivée des conquérants, les sources historiques sont tout à fait vérifiables, surtout quand on sait que, face à la supériorité militaire de l'ennemi, Samori utilisait la tactique de la terre brûlée. Mais aucune source historique digne de ce nom n'atteste que le Roi Djigui Keïta ou que son royaume ait existé à plus forte raison que celui-ci tienne tête au grand empereur Samori. Dans ces conditions, tout se passe comme si l'œuvre romanesque chez Kourouma mettait en lumière la connexion entre la réalité historique et son affabulation romanesque.

\section{Discours crypté, transparence référentielle}

La fiction permet donc d'aller au-delà des préconstruits culturels alors que l'histoire se limite à les reproduire. L'œuvre romanesque de Kourouma entretient constamment l'ambiguïté dans la valeur référentielle des faits racontés. Dans Allah n'est pas obligé, par exemple, si le personnage de l'enfant-soldat et le récit de son histoire relèvent de la fiction, l'écrivain fait référence à des espaces géographiques et à des acteurs politiques vivants. Nous allons observer comment l'écrivain procède au cryptage de l'espace et des personnes tout en laissant dans le récit des indices de décryptage. 
Dans En attendant le vote des bêtes sauvages, le voyage initiatique de Koyaga est raconté au cours de la veille IV. Sur le conseil d'une vieille sorcière, Koyaga se rend à l'école des mâ̂tres de l'autocratie (171). Chaque autocrate a droit à sa geste et chaque geste introduit une boucle narrative. Il rend visite successivement à Tiékoroni, le sage de l'Afrique (176-179), ensuite à Bossouma, empereur du pays des deux fleuves (197-199), après à l'homme au totem de léopard, président du grand fleuve (213-221) et enfin au Roi des Djebels (242-250). En retraçant le parcours de Koyaga, le roman aborde l'histoire de l'Afrique de l'Ouest, du Nord et du Centre. Le pays du président dictateur Koyaga s'appelle la république du Golfe. Cette tournée africaine de chaque nouveau venu au pouvoir est à la fois une coutume de courtoisie et un calcul politique. Comme le souligne si bien le Sora, cet apprentissage devient le motif d'une tournée initiatique car lorsqu'un individu parvenait au pouvoir par la force, il faisait aussitôt la tournée des dictatures pour connaître les ficelles du métier. Cette tournée du nouvel arrivant avait pour but de se faire reconnaître auprès de chefs d'État influents susceptibles de l'introduire dans le cercle, surtout dans les organisations africaines. Il s'agit en général d'un voyage qui a pour motif inavoué la recherche d'une légitimité pour tout président arrivé au pouvoir par coup d'État.

La république des Monts (77) est présidée par l'homme en blanc au totem de lièvre. Ce président s'appelle Nkoutigui Fondio (153). Il a résisté à De Gaulle. Ces indices montrent bien que le narrateur parle de Sékou Touré qui dirige la Guinée, seul pays à voter NON au projet de communauté franco-africaine proposé par le Général De Gaulle en 1958. Dans le récit de la veillée IV le narrateur présente l'homme en blanc, appelé ainsi en vertu d'un lien métonymique parce que Sékou Touré s'habillait toujours en blanc, symbole de la pureté et de la franchise. Le narrateur indique qu'en disant Non à la communauté! Non à la France! Non au néocolonialisme (154), Nkoutigui Fondio avait fait le mauvais choix. Son totem lièvre montre bien qu'il incarne un personnage malin. Cela semble corroborer le fait qu'il avait établi un régime socialiste, ce qui ne l'avait pas empêché de torturer, d'emprisonner, de mentir et d'être un dictateur orgueilleux et impénitent (156-162).

Dans la veillée III, celle qui parle de Maclédio, le Sora indique que le responsable Suprême de la république des monts est auteur de 40 volumes, ses œuvres complètes, dit-il, constituent un gros paquet qu'il offre à ses visiteurs. On sait habituellement que Sékou Touré offrait à tous ceux qui lui rendaient visite des exemplaires de livres appelés les œuvres du PDG ou 
Tomes. Le Sora précise dans son récit que les lignes griffonnées par cet insomniaque versificateur médiocre [en opposition avec Senghor] que les services de la présidence qualifiaient de poésies et de pensées étaient les seuls à être lus étudiés et commentés dans les écoles, instituts et université de la république des monts $(160)^{13}$. Ce passage est en adéquation complète avec l'atmosphère d'effervescence idéologique qui a régné pendant les 26 années de pouvoir de Sékou Touré. Le récit du Sora stipule d'ailleurs que $L a$ connaissance par coeur des ouvres de l'homme en blanc n'était considérée nulle part en dehors de la république des monts comme un savoir. (168) ${ }^{14}$. Tout ce passage s'accorde bien avec l'observation de Barry (2003 : 211) qui souligne que pour ajuster les instruments de la symphonie, le travail d'orchestration mit en réseau le Bureau des Presses de la présidence de la République, qui était chargé de mettre en forme les discours du leader du PDG et de rédiger des additifs. À la suite de quelques réajustements, on obtenait une cuvre collective, dont la forme reliée et publiée portait le nom de Tome. On observe en passant que Mouralis (1987) n'avait certainement pas tort d'accuser le leader guinéen de scribomanie. (Barry 2003 : 211) ${ }^{15}$. Il n'est pas inintéressant de préciser que dans Les Soleils des indépendances, la Guinée, république des monts s'appelle la république de Nikinaï.

Le Sora fait aussi référence à la république des Ebènes (173), pays des Ebènes dans Les Soleils des indépendances, dont le président Tiékoroni, c'està-dire, le vieux petit bonhomme en Maninka, a pour totem le caïman (la plus ancienne des bêtes terrestres). À ce président sont attribués plusieurs pseudonymes dont le bélier du Fasso, [fasso signifiant la patrie], le sage de l'Afrique, l'homme au chapeau mou. Les références géographiques se rapportant à ce pays dont la lagune qui entoure la capitale (173), le palais, la basilique (175) édifiée à Fasso, c'est-à-dire dans le village natal du président, en pleine brousse et même le lac de caïman, tous ces référents aident à montrer qu'il s'agit bien du pays d'Ahmadou Kourouma. Malgré tout, le nom du président ivoirien n'est jamais mentionné explicitement sauf dans Allah n'est pas obligé, où le narrateur le décrit ironiquement de la manière suivante : Ce sage s'appelle Houphouët Boigny. C'est un dictateur, un respectable vieillard blanchi et roussi d'abord par la corruption, ensuite par l'âge et beaucoup de sagesse. (179).

13 Pour plus d'information se référer à : "L'école : d'institution d'enseignement en centre d'éducation révolutionnaire ", op. cit., p. 168-181.

14 Alpha Ousmane Barry, ibidem.

15 Alpha Ousmane Barry, Parole futée \& Peuple dupé, op. cit. 
Le pays des deux fleuves sur lequel règne l'empereur Bossouma, l'homme au totem hyène est aussi une autre république qu'on peut décrypter par le mot empereur qui rappelle Bokassa dont la forme parodiée est Bossouma, c'est-à-dire la puanteur du pet. Le portrait de ce président est encore plus caricatural que celui des autres, car l'homme est surnommé respectivement le gros vin rouge, ce qui laisse penser à des vices liés à la boisson, au portail caparaçonné de décorations, qui indique sa mégalomanie. Il ne résiste pas à la boisson ni au sexe, grotesque, il est méprisé par ses pairs. Cette description dépeint la démesure, qui correspond au symbole de son totem : la sottise. Parlant de lui, le Sora s'exclame Empereur... Empereur! Une vraie honte pour l'Afrique entière! Un saoulard!... Un salaud qui prétend être chef d'État ayant le grade le plus élevé parce qu'il s'est proclamé Empereur. C'est un simple d'esprit (225).

À l'opposé de ce personnage démesuré, Tiékoroni est présenté comme un vieillard rusé, sage et intelligent. Mais le récit le dépeint sous un tableau ambivalent, le présentant sous le voile d'un artiste qui sait transformer les équivoques en ambiguïtés (183). Un homme extrême dans la vertu et le vice, un sac de contradictions. Un homme à la fois généreux comme le fondement d'une chèvre et rancunier, mesquin, méchant comme un pou, un pian; menteur et fabulateur comme une fermme adultère et véridique et entier comme un chasseur de fauves; cruel comme un chat rassasié [...] et tendre comme une poule avec les pintadeaux qu'elle a couvés (191). Comme cela se voit, tout se déploie selon une vision contrastée qui s'apparente bien à celle du Roi du pays des Djebels (241), commandeur des croyants. Il est dépeint également sous le signe de l'intelligence, et de l'habileté politique. Son régime respectable est assis sur une base multiséculaire (241). Malgré le crédit respectable qu'on lui accorde, le Roi du pays des Djebels est aussi moyenâgeux, barbare, cruel, menteur et criminel que tous les autres pères de la nation [...]. Par cette généralisation le récit montre que l'Afrique est de loin le continent le plus riche en pauvreté et en dictature (354). L'ironie sarcastique qui se lit au travers de tout le discours romanesque dans son ensemble est tissée dans un langage indirect, feinte de ce qu'il signifie effectivement.

Le pays des Djebels et du sable est aussi facile à décrypter dans la mesure où le récit indique qu'il s'agit d'un pays musulman du nord (241), gouverné par un musulman blanc, un chef d'état moderne issu d'une dynastie au pouvoir depuis des siècles. Tout comme dans l'extrait précédent, le choix du lecteur porte indubitablement sur le Maroc, même si le récit joue sur un langage contradictoire en apparence, d'où se dégage une vision ironique. 
Deux comparaisons tracent une ligne de conduite discursive qui enracine le récit dans l'histoire politique africaine. La première comparaison établit un parallèle entre Nkoutigui et Tiékoroni de la manière suivante, En réalité, dans l'Afrique des dictatures, Nkoutigui et Tiékoroni, le rusé vieillard, étaient les deux potentats qui, tout en étant différents dans la forme, se ressemblaient le plus dans la façon d'agir (162). Audelà des deux dictateurs, le récit vise à présenter le clivage politique du monde en deux blocs : l'un modéré dont Tiékoroni est le digne représentant et l'autre révolutionnaire qu'incarne Sékou Touré. Tiékoroni n'avait pas la foi, mais il était catholique quant à Nkoutigui, il était musulman. L'homme en blanc croyait aux paroles, aux hommes et au nègre [...] Le rusé aristocrate Tiékoroni ne croyait pas aux paroles, à l'homme et surtout pas au Nègre [...] En cultivant la connivence avec son auditoire, le narrateur demande à celui-ci : sais-tu qui, en définitive eut raison et gagna? C'est Tiékoroni, le rusé petit vieux au chapeau mou [répond-il].

L'ascendant de Tiékoroni sur Nkoutigui s'explique par le fait que Tous les affamés de la République des Monts, tous les affamés de l'Afrique de l'Ouest se dirigent vers la République des Ebènes de Tiékoroni, terre de paix et d'accueil des réfugiés. On ne vit aucun homme de la République des Ebènes voulant rallier la République des Monts, le pays de la dignité du Nègre (163). On sait que dans Les Soleils des indépendances déjà, le récit fait état de l'existence de Dahoméens, de Burkinabé, de la fuite de Diakité par une nuit de clair de lune devant les violences de la révolution dans le pays de Nikinaï. Aussi, la réussite économique de la Côte d'Ivoire en avait fait un pays d'accueil de plusieurs Africains surtout ceux de l'Ouest. À ce niveau, on peut dire que le ton du récit reproduit la réalité par rapport à la situation sociale, politique et économique.

L'ancrage du romanesque dans le réel objectif est observable, comme nous venons de le montrer au niveau du parallèle entre Tiékoroni et Nkoutigui Fondio qui ont entretenu des relations dominées par un conflit datant de la naissance du RDA. Par contre, même si le récit précise que Nkoutigui Fandio et Senghor sont rivaux en poésie et en politique, car le socialisme de l'un s'oppose au libéralisme de l'autre, la fiction prend le dessus avec l'incursion dans le récit d'un troisième personnage, Maclodio. Celui-ci a, dit-on, entretenu des relations de connivence avec Nkoutigui Fondio au cours d'une nuit surprenante de poésie. Les deux interlocuteurs éteignirent toutes les lampes et dans les ténèbres, échangèrent des propos qu'aucun des deux ne révéla jamais (160). Cet épisode mystérieux, qui rappelle un rite d'initiation est digne d'un récit surréaliste. On peut en déduire que le 
narrateur raconte le récit sous le mode fictionnel en alternant des scènes vraisemblables avec des scènes qui relèvent de la pure fiction.

Un autre dictateur qu'on peut ranger dans la catégorie du cryptage concerne le pays du grand fleuve, dont le président est dénommé l'homme au totem de léopard (212), un potentat de la pire espèce. La symbolique des animaux s'apparente énormément aux fables animalières. La panthère est perçue comme un animal féroce, d'ailleurs les mânes (227) le surnomme le grand guerrier qui triomphe de tous les obstacles. Ce portrait ironique et féroce fournit des indices qui laissent penser à Mubutu. D'une part, la désignation de chaque dictateur par un animal en vertu d'un lien métaphorique ou métonymique installe dans le récit le rapport à un mythe fondateur qui unit la famille du président désigné à l'espèce animale, d'où le totémisme; d'autre part, cette symbolique s'inscrit dans le cadre du rapprochement entre la chasse et la politique, ce qui présuppose le franchissement de la barrière qui fonde la différence entre l'humanité et la bestialité, d'où la violence sous toutes ses formes qui exerce son empreinte sur le récit.

Nous venons certes de présenter quelques aspects du cryptage discursif qui concerne essentiellement des référents humains, des personnages historiques. Mais il faut observer que l'ancrage du roman dans la culture africaine est à la base d'un cryptage plus en profondeur du discours romanesque. La connaissance de la culture de l'écrivain est nécessaire à la compréhension de l'épaisseur idéologique du roman africain. Notre analyse précédente est parcellaire par rapport aux divers aspects plus complexes du cryptage. Outre ce discours crypté que les divers indices aident à désambiguïser, plusieurs acteurs politiques africains ou du monde contemporain font leur entrée dans le récit par la grande porte puisqu'ils sont nommés sans aucun cryptage. Ne participant pas directement au déroulement du récit au premier plan, ils sont introduits, non pas comme personnages de l'intrigue, mais comme objets du discours romanesque, ce qui donne à l'intrigue un aspect arborescent et touffu.

\section{Conclusion}

Comme on vient de l'observer tout au long de notre analyse, l'œuvre littéraire francophone d'Afrique est le lieu de représentation de la parole et de la pensée de l'auteur mais aussi d'un corps social plus complexe dont il actualise et modalise les tensions. Elle s'inscrit dans un mouvement énonciatif à l'intérieur d'un discours parallèle au procédé narratif. Ainsi 
l'écriture s'identifie-t-elle à l'espace de la mémoire collective. C'est pourquoi l'acte d'écrire revient à récupérer le geste de la littérature occidentale et à l'adapter à la structure des langages d'un univers culturel différent. L'écriture convoque les acquis culturels africains et les procédés scripturaux occidentaux pour créer une œuvre métissée. Ce double visage de la littérature francophone d'Afrique et les modalités de l'écriture romanesque doivent inciter le chercheur à renouveler en profondeur sa démarche méthodologique.

Dans la tentative de recentrer le travail de recherche sur son objet, l'étude de la littérature francophone d'Afrique est à inscrire dans une sémiotique générale qui se propose de rompre le cloisonnement entre les disciplines. Cela présuppose un déplacement de la question de la norme en vue d'investir celle plus importante de la généricité. L'auteur francophone est souvent un passeur de langue, car la création romanesque maintient la tension entre la langue d'origine et le français. Il rompt ainsi la norme linguistique afin de forger un langage propre dans la mesure où les catégories poétiques thématiques et stylistiques habituelles sont remises en question. L'originalité de l'univers verbal créé est manifeste par ce voyage merveilleux dans l'interlangue dont les traits les plus remarquables s'observent dans la création lexicale de néologismes savoureux. Comme nous l'avons observé tout au long de cette réflexion, l'interlangue est une véritable forme de subversion de la domination symbolique européenne. Images fortes, tours syntaxiques étrangers, vocabulaire exotique, formes métaphoriques évocatrices, permettent de saisir le miroitement d'une vision du monde autochtone. En récupérant les substrats du mythe, de la légende, de l'épopée, du conte, des proverbes, etc. l'interlangue véhicule de nombreuses images qui sont empruntées au fond culturel primordial. La mise en scène de l'oralité assure la vivacité du récit qui se complète d'un rythme narratif singulier décelable dans les répétitions, les parallélismes de construction et les formes accumulatives. On peut donc parler de transcendance textuelle du texte, car l'écriture francophone repose sur un mimétisme discursif. Le discours littéraire reflète l'hétéroglossie du discours social, ce qui veut dire que le texte littéraire ressemble à une composition florale riche de la couleur de toutes ses composantes essentielles. Surplombant la diversité du discours social, l'écriture francophone d'Afrique résulte d'un effet de couture, elle est l'expression la plus complète de l'imaginaire africain ombiliqué au canon de la pensée occidentale. 


\section{Bibliographie}

Angenot, Marc (1988), "Rhétorique du discours social », Langue française, $\mathrm{n}^{\circ} 79$, p. 24-36.

Angenot, Marc (1989), 1889 un état du discours social, Longueuil, Le Préambule.

Barry, Alpha Ousmane (2002), Pouvoir du discours \& discours du pouvoirL'art oratoire chez Sékou Touré de 1958 à 1984, Paris, L'Harmattan.

Barry, Alpha Ousmane (2003), Parole futée \& peuple dupé - Discours et révolution chez Sékou Touré, Paris, L'Harmattan.

Barry, Alpha Ousmane (2004), "Les configurations discursives dans Allah n'est pas obligé d'Ahmadou Kourouma ", in L'intertextualité, Langages et Significations, (CALS), Université de Toulouse Le Mirail, p. 51-58.

Barry, Alpha Ousmane (2004), « La critique sociale et politique dans l'œuvre d'Ahmadou Kourouma ", in L'arbre à Palabres Culture et Développement, $\mathrm{n}^{\circ} 16$, p. 19-56.

Barry, Alpha Ousmane (2006), "Pour une sémiotique trans-culturelle de l'écriture francophone d'Afrique " à paraitre in Actes du colloque de l'AUF, Dakar, Sénégal p. 15-27.

Borgomano Madeleine (2000), Des hommes ou des bêtes? Lecture En attendant le vote des bêtes sauvages d'Ahmadou Kourouma, Paris, L'Harmattan.

Bakhtine, Mikhaîl (1981), Le Principes dialogique suivi des écrits du cercle de Bakbtine, Paris, Seuil.

Eco, Umberto (1985), Lector in fabula..., Presses Universitaires de France. Semen 8, (1993), Configuration discursives, Annales littéraires de l'Université de Besançon, Paris, Les Belles Lettres.

Semujanga, Josias (1999), Dynamique des genres dans le roman africain, Paris, L'Harmattan.

Tine, Aliou (1981), « Pour une théorie de la littérature africaine », Présence Africaine, $\mathrm{n}^{\text {os }} 133-134$, p. 99-121. 